\title{
Preface: Intelligent interactive data visualization
}

\author{
Barbara Hammer • Daniel Keim • Neil Lawrence • Guy Lebanon
}

Received: 18 March 2013 / Accepted: 19 March 2013 / Published online: 13 April 2013

(C) The Author(s) 2013

The increasing amount and complexity of electronic data poses problems for users who analyze that data. They cannot rely on fully automatic techniques for data analysis and visualization, because effective modeling requires an iterative interaction between computerized processing and human analysis. Such a human-in-the-loop approach enables users to interactively refine their hypotheses and modeling assumptions and arrive at conclusions that are impossible for the computer to reach on its own. Intelligent data visualization and its interaction with traditional machine learning serve a central role in this process.

The aim of visual analytics is to develop intelligent interactive visualizations of data. Visual analytics intersects machine learning, intelligent systems, pattern analysis, visualization, computer graphics, and human computer interaction. As such, diverse fields are tackled ranging from the design and evaluation of intuitive data interfaces, efficient and effective data visualization algorithms, evaluation measures and experiment design for user studies, up to software systems for dedicated application areas such as bioinformatics. In this special issue, a variety of novel approaches of machine

B. Hammer $(\varangle)$

CITEC Centre of Excellence, Bielefeld University, Bielefeld, Germany

e-mail: bhammer@techfak.uni-bielefeld.de

D. Keim

Department of Computer and Information Science, University of Konstanz, Konstanz, Germany e-mail: Daniel.Keim@uni-konstanz.de

N. Lawrence

Department of Computer Science, University of Sheffield, Sheffield, UK

e-mail: N.Lawrence@dcs.sheffield.ac.uk

G. Lebanon

College of Computing, Georgia Institute of Technology, Atlanta, USA

e-mail: lebanon@cc.gatech.edu 
learning and intelligent systems is presented to cope with challenges in the emerging area of visual analytics. Out of seventeen submissions, six high quality paper have been accepted, covering diverse aspects in this domain.

The contribution 'On studying a 3D user interface for OLAP' by Sébastien Lafon, Fatma Bouali, Christiane Guinot, and Gilles Venturini addresses interfaces for online analytical processing (OLAP). Unlike standard approaches such as dynamic crosstables, a 3D interface based on virtual reality is proposed which integrates relevant aspects of recent 3D tools. This way, the suitability of modern 3D environments in the context of OLAP can be investigated. Interestingly, however, a user study shows that neither 3D versus 2D nor stereoscopy as opposed to standard 3D displays leads to significant performance yet. An improved visualization of high dimensional data by means of modern nonlinear dimensionality reduction techniques is the focus of the article 'Cartogram Visualization for Nonlinear Manifold Learning Models' by Alfredo Vellido, David L García, and Àngela Nebot. Here, it is argued, that recent non-linear dimensionality reduction (NDR) techniques come at the price of more complex models, in particular complex errors, which are usually not intuitively interpretable for the human observer. To close this gap, the contribution proposes an intuitive way to measure and visualize such errors by means of cartograms, and it verifies its suitability in connection to the generative topographic mapping, as one prominent modern NDR technique, by means of user studies.

The interactive visualization of two specific prominent data structures lies in the center of the next two contributions: the techniques deal with frameworks how to interactively process spatio-temporal data and dynamic networks, respectively. In the contribution 'A Visual Analytics Framework for Spatio-temporal Analysis and Modelling' by Natalia Andrienko and Gennady Andrienko, a visual analytics framework is proposed which enables practitioners to built time-series models for large heterogeneous spatio-temporal data sets in an interactive data-driven way. Building on existing time series modeling tools and clustering tools, interactive grouping, modeling, and model evaluation of time series is supported. The suitability of the approach is demonstrated in the context of two real-life heterogeneus spatio-temporal data sets. The contribution ‘A Regularized Graph Layout Framework for Dynamic Network Visualization' by Kevin Shuai Xu, Mark Kliger, and Alfred Hero centers on dynamic graph layouts in an online scenario; here the challenge is to provide a useful visualization of a given graph such that the layout is similar to a previous image with respect to node locations and grouping. The authors propose a cost function based approach and demonstrate its suitability in the context of two concrete graph drawing schemes.

Finally, two contributions address visual analytics toolkits which are dedicated to specific relevant application domains: hyperspectral image analysis, on the one site, and systems biology data, on the other. Both domains constitute highly relevant and rapidly emerging fields where complex digital data become more and more available due to a dramatic improvement of the underlying sensor technologies and experimental setups. In consequence, researchers and practitioners face huge amounts of complex data which need to be interpreted in an interactive way. The contribution 'Generic Visual Analysis for Multi- and Hyperspectral Data' by Björn Labitzke, Serkan Bayraktar, and Andreas Kolb, provides a system for the interactive analysis of hyperspectral data as they occur e.g. in remote sensing or multispectral imaging. 
Here, a special focus is laid on efficient realizations of linear unmixing algorithms and endmember extraction, to allow an instantaneous decomposition of the images into meaningful components and its interpretation. Systems biology data lies in the centre of the contribution 'Visualizing dimensionality reduction of systems biology data' by Andreas Lehrmann, Michael Huber, Aydin Can Polatkan, Albert Pritzkau, and Kay Nieselt. Here, many popular linear and nonlinear dimensionality reduction techniques are integrated into the framework SpRay which allows the interactive inspection of biological data such as time series of high-dimensional microarray data. Besides two novel visualization techniques which are dedicated to the specific data at hand, the usability of the framework is demonstrated in the context of two real life data sets.

The contributions contained in this special issue constitute spotlights onto relevant research directions in the field of visual analytics, covering interfaces, algorithms, and application oriented systems. The guest editors hope, that these contributions open the way towards further developments and research in this exciting research field. The guest editors would like to thank all authors for contributing to this special issue and all reviewers for their competent judgements and suggestions in this interdisciplinary field. 